Thermophysics and Aeromechanics, 2019, Vol. 26, No. 3

DOI: $10.1134 / \mathrm{S} 0869864319030041$

\title{
Turbulent transport in a swirling jet with vortex core breakdown. PIV/PLIF-measurement and numerical simulation"
}

\author{
A.S. Lobasov ${ }^{1,2}$, V.M. Dulin ${ }^{1,2}$, Ar.A. Dekterev ${ }^{1}$, and A.V. Minakov ${ }^{1,3}$ \\ ${ }^{1}$ Kutateladze Institute of Thermophysics SB RAS, Novosibirsk, Russia \\ ${ }^{2}$ Novosibirsk State University, Novosibirsk, Russia \\ ${ }^{3}$ Siberian Federal University, Krasnoyarsk, Russia \\ E-mail: Alexey.Lobasov@gmail.com
}

(Received November 29, 2018; revised December 3, 2018; accepted for publication December 11, 2018)

Paper reports on optical diagnostics and numerical simulation of the flow structure and transport of a passive scalar in a turbulent swirling jet with vortex core breakdown. Based on the measurements of the instantaneous velocity and concentration fields by PIV and PLIF techniques, the Reynolds stresses and Reynolds fluxes are evaluated and compared to those obtained from URANS and LES simulations. Based on the experimental data and LES-simulation results, the local convective and turbulent transport of the passive scalar are analyzed.

Keywords: swirling turbulent jet, vortex breakdown, turbulent transport, optical methods for flow diagnostics, CFD simulation of turbulent flow.

\section{Introduction}

In combustion chambers of modern gas turbines, a flow swirl is often used for stabilization of flames. The flow swirl intensifies mixing, provides stable combustion in a compact volume with high ignition efficiency and flame stability in a wide range of the fuel-air ratios [1-3]. For jets, the imposed swirl promotes heat and mass transfer in the initial region of the flow [1, 4-6]. At the same time, the structure and dynamics of swirling jets is much more complex in comparison to non-swirling jets due to a number of effects, viz., formation a wake region at the jet axis, vortex core breakdown and formation of a central recirculation zone when the swirl rate exceeds a certain critical value, presence of helical vortex structures, including spiraling precessing vortex core [1, 7-15].

Modern methods of numerical simulation allow for a fast optimization of jet-burners and combustion chambers [16]. However, reliable numerical modelling of complex unsteady processes in combustion chambers with strong swirl require complex experimental data for verification of the used mathematical models. The problem of numerical simulation methods perfection for swirling turbulent jets without combustion is relevant for the last 50 years [17].

\footnotetext{
* The work was financially supported by the Russian Science Foundation (Grant No. 16-19-10566).
}

(C) A.S. Lobasov, V.M. Dulin, Ar.A. Dekterev, and A.V. Minakov, 2019 
The literature presents extensive data on flow structure of turbulent swirling jet, and the comprehensive review is available in [18]. However, these data are limited by average velocity and pressure profiles, correlation functions and spectra of turbulent fluctuations [19]. Moreover, the structure of swirling flows is strongly sensitive to density gradients, that significantly complicates studies of heat transfer. In particular, the buoyancy can change conditions for vortex breakdown and recirculation zone shape [20].

Development of optical measurement techniques, such as Laser Doppler Anemometry (LDA) and Particle Image Velocimetry (PIV), allows to study in detail turbulent transport in swirling jets [21-24], including measurements of the Reynolds stresses and third-order moments of velocity fluctuations. Besides, almost non-intrusive measurements increase the reliability of the data. The Planar Laser-induced Fluorescence (PLIF) technique provides almost non-intrusive measurements of the spatial distributions of concentration [25-27]. However, experimental data on concentration distributions in swirling turbulent jets measured by PLIF are practically absent in literature.

The aim of this paper is to study experimentally the transport of passive scalar in the near field of a swirling turbulent jet with vortex breakdown and analyze the efficiency of popular numerical simulation methods based on solving the Unsteady Reynolds-Averaged Navier-Stokes equations (URANS) and Large-Eddy Simulation (LES) to predict the flow and transport.

\section{Methods}

The object of the study is a swirling jet issued from the axisymmetric contraction nozzle (the outlet diameter was $d=15 \mathrm{~mm}$ ) with vane swirler mounted inside (see Fig. 1 and detailed description in [24]). The swirl rate $S$ is determined from the geometric parameters of the swirler [1]:

$$
S=\frac{2}{3}\left(\frac{1-\left(d_{1} / d_{2}\right)^{3}}{1-\left(d_{1} / d_{2}\right)^{2}}\right) \operatorname{tg}(\psi),
$$

here $d_{1}=7 \mathrm{~mm}$ is the diameter of centerbody supporting the vanes, $d_{2}=27 \mathrm{~mm}$ is the external diameter of the swirler, and $\psi$ is the vanes inclination angle. The swirler used in the current research (with inclination angle $\psi=55^{\circ}$ ) provides a swirl rate $S=1.0$. This swirl rate exceeds the critical value $(\approx 0.6)$ for the vortex breakdown in jet flows (see $[14,15,20,28-30])$.

The flow was supplied from a pressure line (pressure up to $20 \mathrm{~atm}$ ), and its flow rate was controlled by mass flow meters (Bronkhorst). An acetone seeder was used to introduce acetone vapor (used as a passive scalar in the present study) into the air flow. The seeder represented a container with liquid acetone, which was placed in a heated water bath to provide thermal stabilization. The air flow was bubbled through the liquid acetone using bypass scheme.

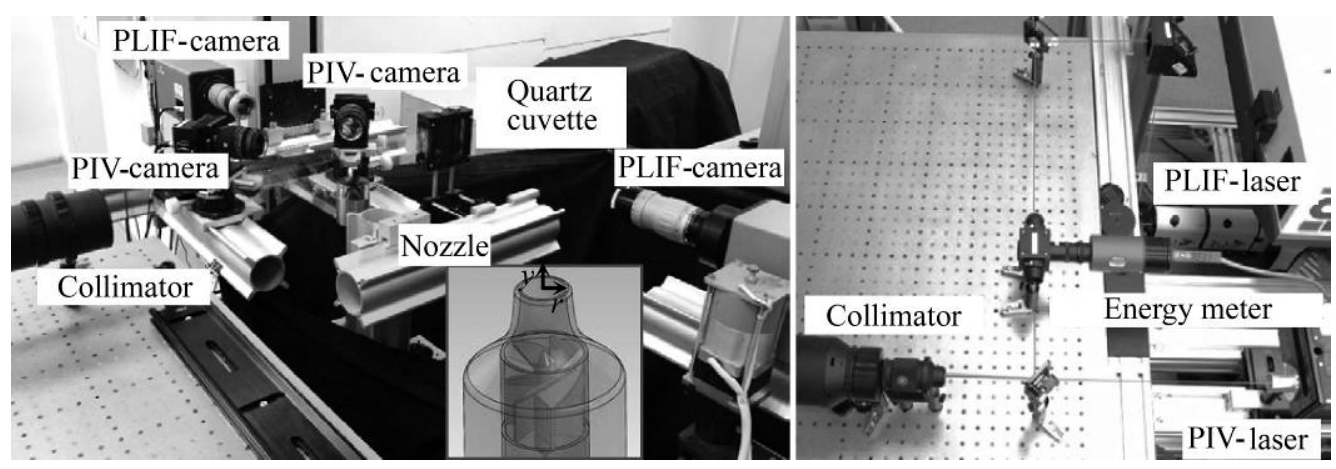

Fig. 1. Photograph of the experimental setup and equipment of PIV/PLIF systems. The geometry of the nozzle is presented by inset. 
The temperature of the air with acetone vapor at the seeder exit was approximately $50^{\circ} \mathrm{C}$. The concentration of acetone vapor in the jet was below $3 \%$. The solid particles of titanium oxide with the size of $0.5 \mu \mathrm{m}$ were used for flow seeding to provide PIV measurements. In order to introduce the tracer particles to the jet flow another seeder (the tank with a volume of 2 liters) with mechanical mixer was used. The surrounding air was seeded by a fog generator (Magnum). The Reynolds number (based on the bulk flow velocity of the jet $U_{0}=5 \mathrm{~m} / \mathrm{s}$, kinematic viscosity of the air, nozzle diameter) was 5000 .

The photograph of the experimental setup is shown in Fig. 1. For the excitation of the acetone fluorescence, the radiation of fourth harmonic of a solid state Nd:YAG-laser (Quantel, Brilliant B) was used. The energy of laser pulse at the wavelength $266 \mathrm{~nm}$ was $60 \mathrm{~mJ}$. To remove residual laser radiation in the PLIF optical system the spectral separation of laser harmonics was implemented by dichroic mirror. After dichroic mirror, the second harmonic of the laser radiation (separated from UV-radiation) was captured by beam dump and the UVradiation was directed into the collimator by system of rectangular prisms. The collimator converts the laser beam into a sheet with the width of $50 \mathrm{~mm}$ and the thickness below $0.8 \mathrm{~mm}$. Approximately $10 \%$ of UV-radiation was directed to the energy meter (LaVision) for control of each laser pulse energy.

The fluorescence of acetone vapor was registered by an image intensifier (with the quantum efficiency of $25 \%$ at the considered wavelength range $290-320 \mathrm{~nm}$ ), which was connected to LaVision ImagerPro sCMOS camera (the resolution of $2560 \times 2160$ pixel and dynamic range of 16 bits) and equipped with a quartz lens (f\#2.8, $100 \mathrm{~mm})$ with a set of optical filters. The filter set includes the Multi-Notch filter which removed the harmonics of Nd:YAG-laser (1064, 532 , and $366 \mathrm{~nm}$ ) and the interference band-pass filter with transmission range from 280 to $600 \mathrm{~nm}$. The using of the optical filters was necessary to remove unwanted intensity of PIV and PLIF lasers, including reflections from the nozzle. The exposure time for PLIF images was 200 ns.

The velocity fields were measured by PIV-system POLIS in a stereo configuration. The tracer particles in the flow were illuminated by the radiation of second harmonic of a double-head pulsed Nd:YAG laser (Quantel, EverGreen). The lasers radiation was spatially aligned into single optical axis with the pulse energy of $53 \mathrm{~mJ}$. The wavelength of PIV laser radiation was $532 \mathrm{~nm}$. The tracer images were captured by two CCD cameras (ImperX IGVB4820), equipped with SIGMA AF $50 \mathrm{~mm}$ lenses (f\#2.8 EX DG Macro) and band-pass optical filters (Edmund Optics) with the transmittance of $60 \%$ at the wavelength $532 \mathrm{~nm}$ and the full width at half maximum of $10 \mathrm{~nm}$. The resolution of each PIV image was $2048 \times 2048$ pixels

The PIV and PLIF systems were synchronized by two programmable timing units (LaVision and POLIS). The frame rate of combined system was $1.5 \mathrm{~Hz}$. The registration of fluorescence of acetone vapor was captured between the first and second PIV laser pulses. The time interval between two PIV laser pulses was $40 \mu \mathrm{s}$. The test of spatial alignment of PIV and PLIF lasers was performed by a photo-sensitive paper, which was placed in the measurement volume. For spatial calibration of PIV and PLIF cameras, the plane calibration target (Edmund Optics) was used. The calibration target was a diffusive white plate with black round dots (the dot's size and distance between nearest dots were $2 \mathrm{~mm}$ ).

For quantitative interpretation of PLIF-signal, several mathematical algorithms were used. The processing algorithms take into account fluctuations of laser energy from pulse to pulse, including the correction of nonuniform spatial distribution of energy in the laser sheet and nonuniform spatial sensitivity of camera sensor. The spatial calibration for the measurement planes was carried out using a third-order model for image projections on the camera's sensors. The parameters of the third-order model were used for reconstruction of the threecomponent velocity field from stereo-PIV images and for spatial alignment of PIV and PLIF images. In the case of calculating the velocity fields the background signal was subtracted. The background signal was calculated by averaging the intensity values in each pixel for the entire set of images. The velocity fields were evaluated by an iteration cross-correlation 
algorithm with continuous image shift and deformation of interrogation area between iterations. The finite size of interrogation area was $32 \times 32$ pixels with overlapping of $50 \%$. The algorithm also takes into account the number of particles in interrogation area (if the number of particles is below five, then the velocity vector was not calculated [31]).

For CFD simulation of turbulent swirling flows, several models were used, including two-equation ( $k-\omega$ SST) and the Reynolds stresses models (RSM LRR) in the frame of URANS approach. In addition, the large eddy simulation (LES) approach with Wall Adapting Local Eddy-viscosity model (WALE) was used. The finite-volume method was applied as discretization procedure to solve the equations of transport. Pressure-velocity coupling for incompressible flow was achieved by the SIMPLEC procedure. The approximation of convective terms of URANS equations were approximated by second-order upwind scheme. In the case of LES approach, for approximation of convective terms, the central difference scheme was used. For approximation of convective terms of turbulence properties equations, the second-order upwind scheme was used. The diffusion terms were approximated with a second-order scheme. The implicit second-order scheme for time integration was applied. The time step satisfied the condition that average $\mathrm{CFL}<2$.

The computations have been carried out using unstructured grids with more than two millions nodes (see Fig. 2). Several layers of prismatic cells were created at the nozzle walls for better resolution of the boundary layer. The widely used Kolmogorov scale, the energy-motion vortex scale, and the shear scale were used as a criterion of sufficient resolution of computational grid [32]. The average distance of the wall-nearest grid nodes normalized by the wall unit, $y_{1}^{+}$, was 1.8. The characteristic cell size $\Delta=(\Delta x \times \Delta y \times \Delta z)^{1 / 3}$ was compared with the Kolmogorov dissipative scale $L_{\mathrm{K}}=\left(v^{3} / \varepsilon\right)^{1 / 4}$ (here $v$ is the kinematic viscosity), the generalized Corrsin "shear" scale $L_{\mathrm{s}}=\left(\varepsilon / S^{3}\right)^{1 / 2}$ (here $\varepsilon$ is the dissipation rate obtained from RSM) and the second invariant of strain rate tensor $S=\left(S_{i j} S_{i j}\right)^{1 / 2}$. For the RSM model, the ratio $\Delta / L_{\mathrm{K}}$ was ranged from 10 to 20 . The ratio of the cell size to shear turbulence scale $\Delta / L_{\mathrm{s}}$ was less 1.0 in the main flow region.

\section{Results}

Measurements of the instantaneous distributions of the passive scalar concentration $c$ and three-component velocity fields $\mathbf{u}=\left(u_{x}, u_{y}, u_{z}\right)$ are performed in a longitudinal cross-section of flow $(x, y)$ passing through the jet axis $(\mathrm{O} y)$. The $\mathrm{O} x$ axis is collinear with the radial direction. For the cross section for $x>0$, the radial and azimuthal velocity components are equal to $u_{x}$ and $u_{z}$. Results of the numerical simulation are interpolated to the same coordinate grid as

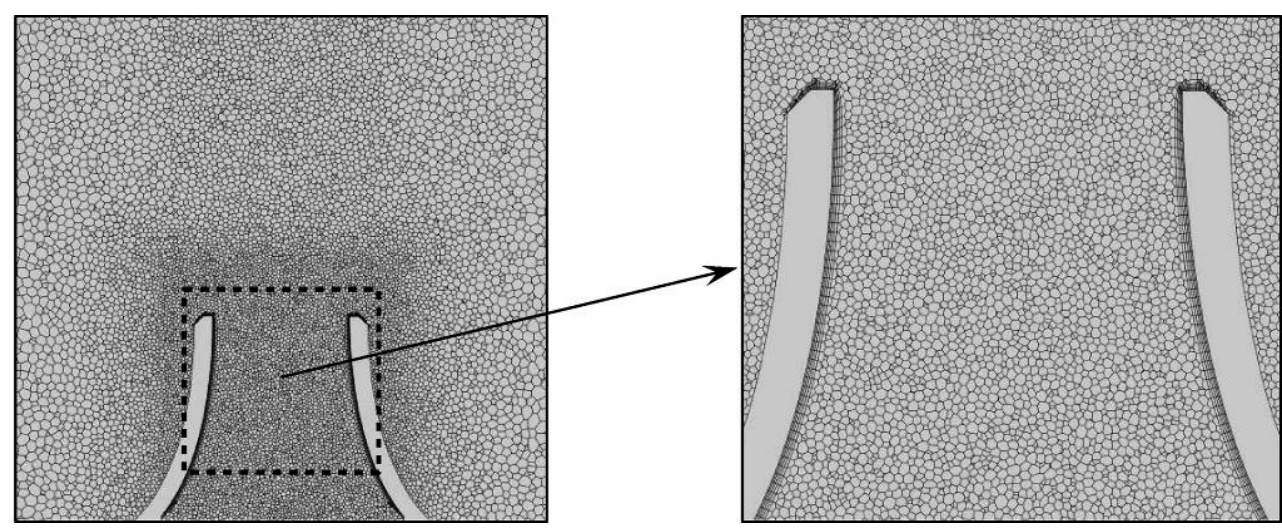

Fig. 2. Example of unstructured computational grid in the nozzle region. 

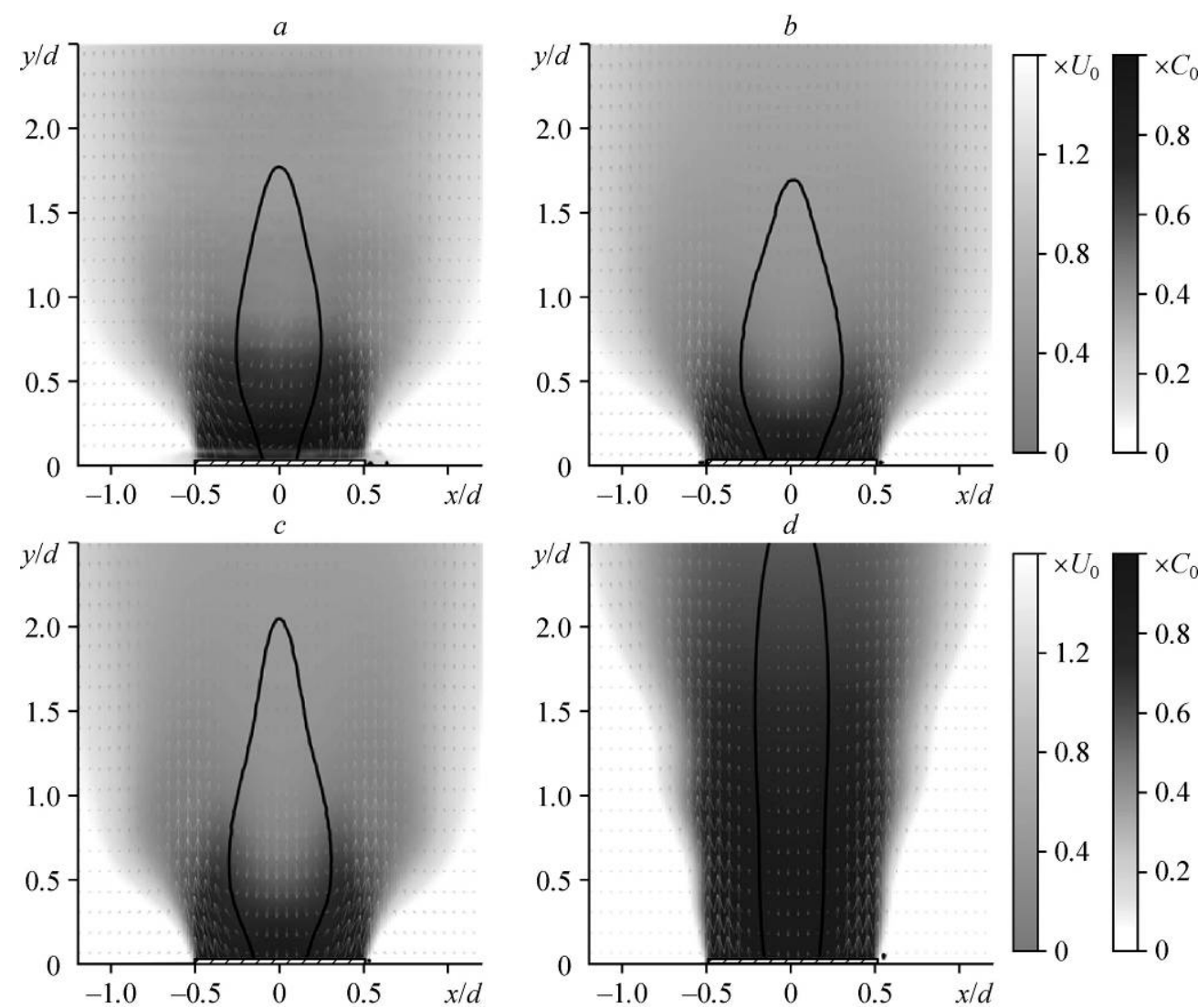

Fig. 3. Time-averaged velocity (vectors) and concentration fields for the swirling turbulent jet.

The solid line surrounds the recirculation zone; $a$ - experiment; numerical simulation: LES $(b)$, RSM $(c)$, SST $(d)$.

the experimental data. The measured and calculated by LES- and URANS-approaches spatial distributions of the mean velocity $\mathbf{U}$ and concentration $C$ for the swirling turbulent jet with vortex breakdown are shown in Fig. 3. The central recirculation zone is visualized by the condition $U_{y}<0$. The solution of SST-model is stationary in contrast to that of the RSMmodel. One can observe that the SST-model does not correctly predict the opening angle of the jet, the shape of recirculation zone, and rate of mixing with the surrounding air. The average characteristics of flow calculated by the LES and URANS RSM approaches demonstrate a good agreement with experimental data.

For the quantitative comparison of the results, Figure 4 demonstrates profiles of the velocity components in cross section $y / d=0.7$, which corresponds to the maximum width of the recirculation zone. Values of the radial velocity component are smaller than $10 \%$ of the velocity vector magnitude. Therefore, the PIV measurements for this component are effected by a bias error. This issue is clearly seen near the jet axis. The comparison between the experimental data and numerical simulation shows that the SST approach with stationary solution does not provide even qualitative prediction of the average concentration distribution. The data calculated by LES and URANS RSM approaches show a good agreement with the distributions of the average velocity and concentration obtained from the experiment (especially in case of the LES approach).

Comparison of local variances of the concentration and velocity fluctuations, Reynolds stress and turbulent flux are shown in Fig. 5 (SST data are not presented). In case of URANS 

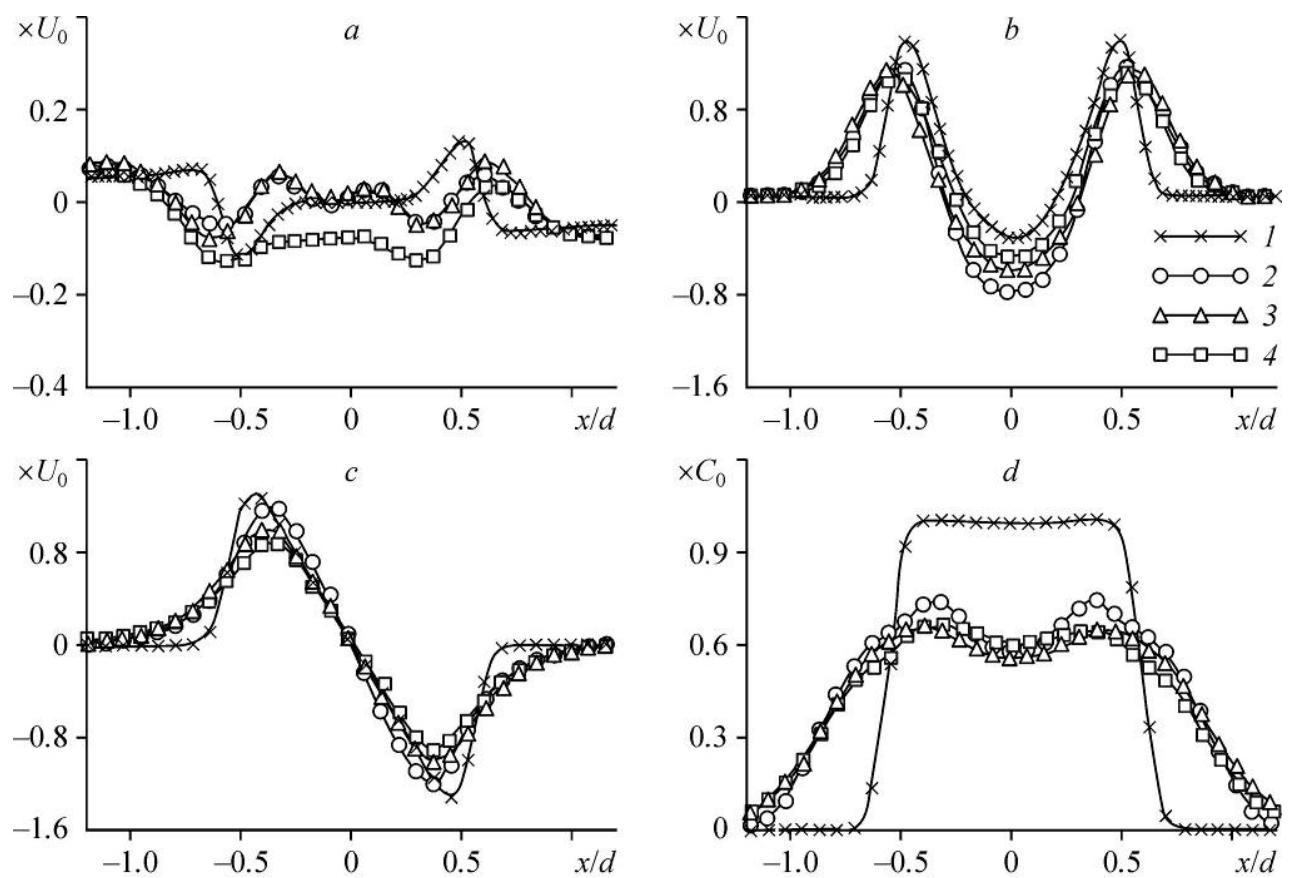

Fig. 4. Profiles of the time-averaged velocity and concentration for the swirling turbulent jet for cross section $y / d=0.7$.

Velocity components $U_{x}(a), U_{y}(b), U_{z}(c)$, concentration $C(d)$; 1 - SST, 2 - RSM, 3 - LES, 4 - experiment.
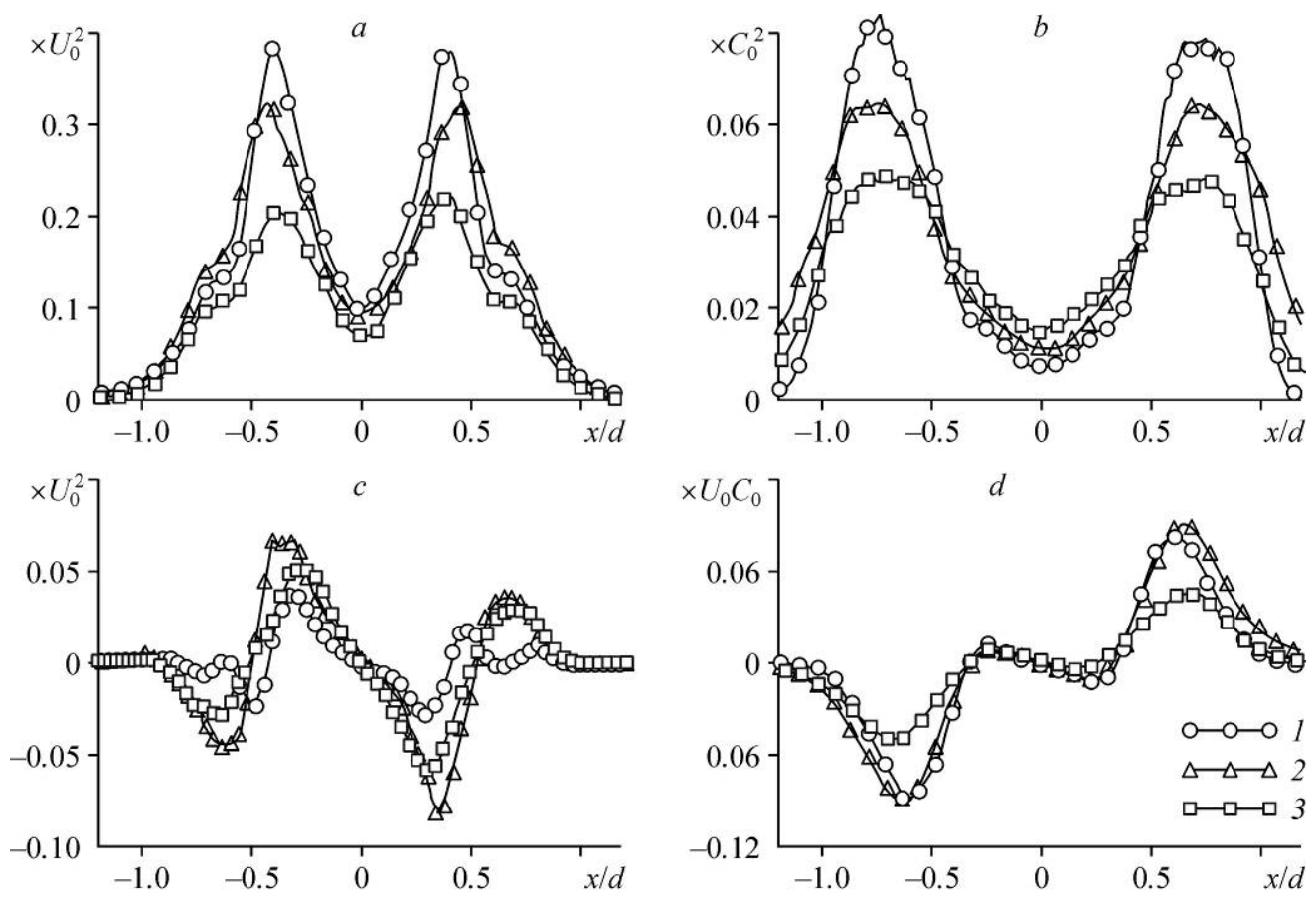

Fig. 5. Profiles of local variance of velocity and concentration, Reynolds stresses and flux for a turbulent swirling jet for cross section $y / d=0.7$.

Flow profiles $\left\langle u_{y}^{\prime} u_{y}^{\prime}\right\rangle(a),\left\langle c^{\prime} c^{\prime}\right\rangle(b),\left\langle u_{x}^{\prime} u_{y}^{\prime}\right\rangle(c),\left\langle u_{x}^{\prime} c^{\prime}\right\rangle(d)$; 1 - RSM, 2 - LES, 3 - experiment. 
RSM approach, the variance of the velocity fluctuations corresponds to a sum of the coherent part (unsteady pulsations) and stochastic part (modelled via RANS approach) fluctuations. The stochastic part of the velocity variance is 5 times lower than the coherent part. One can observe that data calculated by LES approach correspond better to the Reynolds stresses $\left\langle u_{x}^{\prime} u_{y}^{\prime}\right\rangle$ measured by PIV. For calculation of the turbulent flux $\left\langle u_{x}^{\prime} c^{\prime}\right\rangle$, the LES and URANS RSM approaches provide similar results. Magnitude of the turbulent flux profile obtained from experimental data is significantly lower. Moreover, in the region of recirculation zone, the variances of the concentration and velocity fluctuations measured by PIV and PLIF are significantly lower than those obtained by numerical simulation. In this region of intensive turbulent pulsations, the underestimation of the variance amplitude may be due to a finite spatial resolution of the PIV and PLIF techniques.

For the analysis of local turbulent transport in the swirling jet with the vortex core breakdown, all term values of the Reynolds-averaged mass transport equation for the passive scalar $c$ have been estimated in the cylindrical coordinate system (2):

$$
\begin{aligned}
& U_{y} \frac{\partial C}{\partial y}+U_{r} \frac{\partial C}{\partial r}+\frac{\partial\left\langle u_{y}^{\prime} c^{\prime}\right\rangle}{\partial y}+\frac{\partial\left\langle u_{r}^{\prime} c^{\prime}\right\rangle}{\partial r}+\frac{\left\langle u_{r}^{\prime} c^{\prime}\right\rangle}{r}=D\left\{\frac{\partial^{2} C}{\partial y^{2}}+\frac{1}{r} \frac{\partial}{\partial r}\left(r \frac{\partial C}{\partial r}\right)\right\} . \\
& \begin{array}{llllll}
1 & 2 & 3 & 4 & 5 & 6
\end{array}
\end{aligned}
$$

The first and second terms describe the transport by the mean flow (advection). The third term and sum of the fourth and fifth terms correspond to the turbulent transport in the axial and radial directions, respectively. The contribution of the sixth term corresponds to molecular diffusion, where $D$ is the molecular diffusion coefficient.

The results of equation terms estimation based on the experimental data and LESsimulation are shown in Fig. 6. For the flow at the considered Reynolds number $(\operatorname{Re}=5000)$, the molecular diffusion is found to be negligibly small. The transport by the mean flow occurs mainly in the axial direction and appears to be compensated by the turbulent diffusion in the radial direction. Intensity of the turbulent diffusion in the axial direction is much lower. To test for reliability of the mass transport equation terms estimation from the experimental data, the sum of all transport terms is evaluated. For the experimental data, it is found to be less than $14 \%$ of the convective transport term magnitude. Hence, the measurements are provided with reliable accuracy.
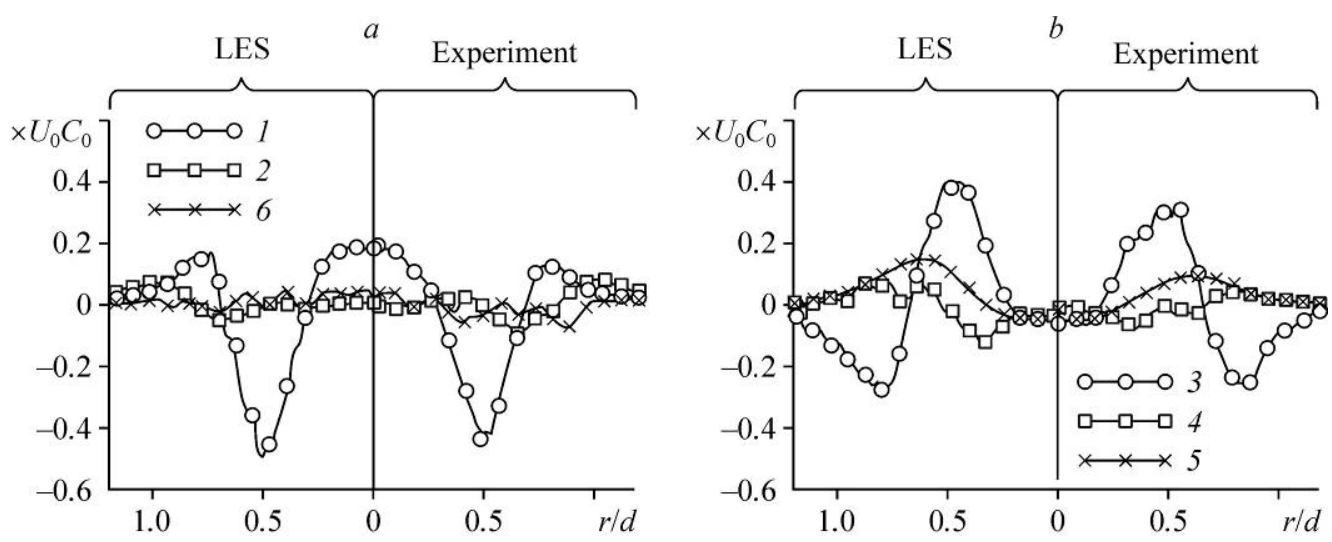

Fig. 6. Terms of transport equation (2) for the turbulent swirling jet for cross-section $y / d=0.7$. Comparison between experimental data and LES-simulation.

$1-6$ correspond to the values of terms $1-6$ in equation (2). 


\section{Conclusion}

Flow structure of the turbulent swirling jet with vortex breakdown has been investigated by using the modern methods of optical diagnostic (PIV and PLIF) and numerical simulation (URANS and LES). Local transport of the passive scalar admixture in the region of recirculation zone has been analyzed. The LES approach is found to provide the most accurate simulation of swirling turbulent jet structure, including turbulent flux and Reynolds stresses. The unsteady RSM approach also shows a good agreement with experimental data and allows one to reliably predict the shape of recirculation zone, opening angle of the jet, and spatial distribution of the passive admixture. The used SST approach provides a practically steady solution, which does not correspond to the structure of the real flow.

Based on the experimental data and LES-simulation, the profiles of different terms of the mean mass transport equation have been obtained for a cross section through the central recirculation zone. The analysis reveals that the molecular diffusion for the flow with $\mathrm{Re}=5000$ is negligibly small. The transport by the mean flow occurs mainly in the axial direction and is found to be compensated by turbulent diffusion in the radial direction. Intensity of the turbulent diffusion in the axial direction is found to be much smaller.

The authors are grateful to D.K. Sharaborin for assistance in experiments.

\section{References}

1. A.K. Gupta, D.G. Lilley, and N. Syred, Swirl flows. England, Tunbridge Wales, Kent: Abacus Press, 1984.

2. R. Weber and J. Dugué, Combustion accelerated swirling flows in high confinements, Progress in Energy and Combustion Sci., 1992, Vol. 18, No. 4, P. 349-367.

3. A.I. Mikhaylov, G.M. Gorbunov, V.V. Borisov, L.A. Kvasnikov, and N.I. Markov, Rabochiy Protsess i Raschet Kamer Sgoraniya Gazoturbinnykh Dvigateley [Workflow and Calculation of Gas Turbine Engine Combustors], Defence Industry Publ., Moscow, 1959.

4. C.P. Zemtsop, M.K. Stöllinger, S.D. Heinz, and D. Stanescu, Large-eddy simulation of swirling turbulent jet flows in absence of vortex breakdown, AIAA J., 2009, Vol. 47, No. 12, P. 3011-3021.

5. F. Cozzi, A. Coghe, and R. Sharma, Analysis of local entrainment rate in the initial region of isothermal free swirling jets by stereo PIV, Experimental Thermal and Fluid Sci., 2018, Vol. 94, P. 281-294.

6. J. Fröhlich, M. García-Villalba, and W. Rodi, Scalar mixing and large-scale coherent structures in a turbulent swirling jet, Flow, Turbulence and Combustion, 2008, Vol. 80, No. 1, P. 47-59.

7. S.V. Alekseenko, V.M. Dulin, Yu.S. Kozorezov, D.M. Markovich, S.I. Shtork, and M.P. Tokarev, Flow structure of swirling turbulent propane flames, Flow, Turbulence and Combustion, 2011, Vol. 87, No. 4, P. 569-595.

8. M.R. Ruith, P. Chen, E. Meiburg, and T. Maxworthy, Three-dimensional vortex breakdown in swirling jets and wakes: direct numerical simulation, J. Fluid Mech., 2003, Vol. 486, P. 331-378.

9. K. Oberleithner, C.O. Paschereit, R. Seele, and I. Wygnanski, Formation of turbulent vortex breakdown: intermittency, criticality, and global instability, AIAA J., 2012, Vol. 50, No. 7, P. 1437-1452.

10. O. Lucca-Negro and T. O'Doherty, Vortex breakdown: a review, Progress in Energy and Combustion Sci., 2001, Vol. 27, No. 4, P. 431-481.

11. H. Liang and T. Maxworthy, An experimental investigation of swirling jets, J. Fluid Mech., 2005, Vol. 525, P. $115-159$.

12. . Billant, J.M. Chomaz, and P. Huerre, Experimental study of vortex breakdown in swirling jets, J. Fluid Mech., 1998, Vol. 376, P. 183-219.

13. T. Loiseleux and J.M. Chomaz, Breaking of rotational symmetry in a swirling jet experiment, Phys. Fluids, 2003, Vol. 15 , No. 2, P. 511-523.

14. S.I. Shtork, O. Comas, E.C. Fernandes, and M.V. Heitor, Aerodynamic structure of unsteady swirling flow downstream of a sudden expansion, Thermophysics and Aeromechanics, 2005, Vol. 12, No. 2, P. 217-228.

15. I.V. Litvinov, D.K. Sharaborin, and S.I. Shtork, Finding of parameters of helical symmetry for unsteady vortex flow based on phase-averaged PIV measurement data, Thermophysics and Aeromechanics, 2015, Vol. 22, No. 5, P. 647-650.

16. W.C. Reynolds, J.J. Alonso, and M. Fatica, Aircraft gas turbine engine simulations, in: 16th AIAA Computational Fluid Dynamics Conference, 2003, P. 1-17.

17. D.G. Lilley and N.A. Chigier, Nonisotropic turbulent stress distribution in swirling flows from mean value distributions, Int. J. Heat and Mass Transfer, 1971, Vol. 14, No. 4, P. 573-585.

18. R.B. Akhmedov, T.B. Balagula, F.K. Rashidov, and A.Yu. Sakaev, Aerodinamika Zakruchennoi Strui [Aerodynamics of Swirling Jet], Energy Publ., Moscow, 1977. 
19. S.Yu. Krasheninnikov, Investigation of a submerged air jet during high-intensity swirling, Fluid Dyn., 1971, Vol. 6, No. 6, P. 1039-1045.

20. D. Mourtazin and J. Cohen, The effect of buoyancy on vortex breakdown in a swirling jet, J. Fluid Mech., 2007, Vol. 571, P. 177-189.

21. S. Komori and H. Ueda, Turbulent flow structure in the near field of a swirling round free jet, Phys. Fluids, 1985, Vol. 28, No. 7, P. 2075-2082.

22. S.V. Alekseenko, A.V. Bilsky, V.M. Dulin, and D.M. Markovich, Experimental study of an impinging jet with different swirl rates, Int. J. Heat and Fluid Flow, 2007, Vol. 28, No. 6, P. 1340-1359.

23. S.V. Alekseenko, V.M. Dulin, Yu.S. Kozorezov, and D.M. Markovich, Effect of axisymmetric forcing on the structure of a swirling turbulent jet, Int. J. Heat and Fluid Flow, 2008, Vol. 29, No. 6, P. 1699-1715.

24. S.V. Alekseenko, V.M. Dulin, Yu.S. Kozorezov, and D.M. Markovich, Effect of high-amplitude forcing on turbulent combustion intensity and vortex core precession in a strongly swirling lifted propane/air flame, Combustion Sci. and Technology, 2012, Vol. 184, No. 10-11, P. 1862-1890.

25. L.K. Su, Measurements of the three-dimensional scalar dissipation rate in gas-phase planar turbulent jets, Center for Turbulence Research Annual Briefs, 1998, P. 35-46.

26. G.-H. Wang, N.T. Clemens, R.S. Barlow, and P.L. Varghese, A system model for assessing scalar dissipation measurement accuracy in turbulent flows, Measurement Sci. and Technology, 2007, Vol. 18, No. 5, P. 1287-1303.

27. R. Örlü and P.H. Alfredsson, An experimental study of the near-field mixing characteristics of a swirling jet, Flow, Turbulence and Combustion, 2008, Vol. 80, No. 3, P. 323-350.

28. N. Syred and J.M. Beer, Combustion in swirling flows: a review, Combustion and Flame, 1974, Vol. 23, No. 2, P. 143-201.

29. P.M. Anacleto, E.C. Fernandes, M.V. Heitor, and S.I. Shtork, Swirl flow structure and flame characteristics in a model lean premixed combustor, Combustion Sci. and Technology, 2003, Vol. 175, No. 8, P. 1369-1388.

30. E.C. Fernandes, M.V. Heitor, and S.I. Shtork, An analysis of unsteady highly turbulent swirling flow in a model vortex combustor, Experiments in Fluids, 2006, Vol. 40, No. 2, P. 177-187.

31. D.M. Markovich and M.P. Tokarev, Algorithms of reconstruction of three component velocity field in the Stereo PIV method, Vychisl. Metody i Programmirovanie, 2008, Vol. 9, No. 3, P. 311-326.

32. F. Picano and K. Hanjalić, Leray- $\alpha$ regularization of the Smagorinsky-closed filtered equations for turbulent jets at high Reynolds numbers, Flow, Turbulence and Combustion, 2012, Vol. 89, No. 4, P. 627-650. 